\title{
An Unusual Case of Iatrogenic ALUM Toxicity as Quack Remedies Resulting in Fulminant Hepatic Failure of Young School Student
}

\author{
Vivekanshu Verma $^{1 *}$, Shagun Walia ${ }^{2}$, Devendra Richhariya ${ }^{3}$ and Neelam Mohan ${ }^{4}$ \\ ${ }^{1}$ Associate Consultant, Emergency and Trauma Care, Medanta - The Medicity, Gurugram, India \\ ${ }^{2}$ Fellow in Paediatric Gastroenterology, Medanta - The Medicity, Gurugram, India \\ ${ }^{3}$ Associate Director, Emergency and Trauma care, Medanta - The Medicity, Gurugram, India \\ ${ }^{4}$ Director, Paediatric Gastroenterology, Medanta - The Medicity, Gurugram, India \\ *Corresponding Author: Vivekanshu Verma, Associate Consultant, Emergency and Trauma Care, Medanta - The Medicity, Gurugram, \\ India.
}

Received: March 18, 2019; Published: May 22, 2019

DOI: $10.31080 /$ ASPS.2019.03.0291

\begin{abstract}

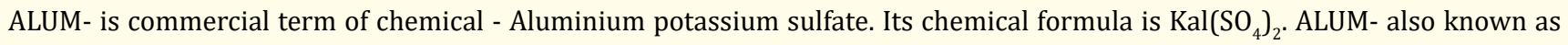
Potash Alum in Indian groceries [1]. Alum is consumed on advice of unqualified quacks as a household remedy for various ailments in villages, due to lack of availability of doctors, Hospitals, pharmacies and allopathic medications in rural countryside. We are presenting a recent unusual case of iatrogenic fatal Alum toxicity in a young minor, in whom Alum was dispensed by a traditional local quack for curing patient's prolonged fever, resulting in drug induced liver injury, leading to fatal complication of fulminant hepatic failure, in absence of no specific antidote of Alum, requiring urgent liver transplantation.

Keywords: Alum; Toxicity; Liver; Aluminium
\end{abstract}

\section{Background}

ALUM is a double salt of sulphate of aluminium and potassium. It is transparent and colourless and is available in octahedral crystals or as a white powder with a sweetish, astringent taste [2]. Alum contains Aluminium, which is a neurotoxin, has been numerously studied, but its hepatotoxic effect is poorly studied. Since Liver is one of the main target organs of aluminium burden and liver is involved in metabolism and detoxification of heavy metals including aluminium, Lead, Cadmium etc. ALUM- is commercial term of chemical - Aluminium potassium sulfate. Its chemical formula is Kal $\left(\mathrm{SO}_{4}\right)_{2}$. ALUM- also known as Potash Alum in market. In Ayurveda, where it is called phitkari or saurashtri. Ayurveda recommends Phitkari (Alum)- in Treatment of Pediculosis Capitis [13]. In traditional Chinese medicine it is called ming fan. White Crystal -Whitener-Tightener Skin-Minimize Facial Hair Growth. It is commonly used in water purification, leather tanning, dyeing, fireproof textiles, and baking powder. It also has cosmetic uses as a deodorant, as an aftershave treatment and as a styptic for minor bleeding from shaving. Alum is medically used in urinary bladder washings to treat hemorrhagic cystitis. Toxic part of Alum is Aluminium, so is the name shortened on its toxic role. Patients with renal insufficiency are at high risk for aluminum (Al) toxicity. Other etiologies of aluminum toxicity include bladder irrigation with alum and extensive use of aluminum -containing antacids. Additional iatrogenic sources of aluminum include total parenteral nutrition solutions and vaccines. Ingested aluminum is not metabolized by the body and is excreted unchanged by the kidney. Target Tissues in Aluminium Intoxication is Brain, Bone, Liver and Kidney.

\section{Case Presentation}

A 16-year-old male school student was brought to our emergency department from nearby village in the early morning hours with recurrent vomiting, abdominal pain and seizures, and a low 
Glasgow Coma Score (GCS) of 8. His physical examination showed icterus, his capillary glucose was $40 \mathrm{mg} / \mathrm{dl}$. Immediately $25 \%$ Glucose was given. He was having recurrent seizures, with past history of fever off and on since one month and history of consumption of 2-3 teaspoons (approx. 20gm) of Phitkari (Alum) last evening on verbal advice of an roadside quack physician in his village to treat his prolonged fever. In ER we secured his airway to manage his compromised airway during ongoing seizure, Patient was immediately sedated, intubated, ventilated and started on antiepileptics. On investigation, his Serum Total Bilirubin was $25 \mathrm{mg} / \mathrm{dl}$ with serum aminotransferase $=5000$ units $/ \mathrm{dl}$. Viral markers were negative, and blood cultures were sterile. Serum Aluminium levels revealed $=17 \mathrm{mg} / \mathrm{l}$ levels on $10^{\text {th }}$ day of ingestion (Normal range $=0-5 \mathrm{mg} / \mathrm{L}$ ). Patient was diagnosed as a case of acute fulminant hepatic failure with neurotoxicity as a result of consumption of toxic dose of Alum- Aluminium potassium sulfate. N-Acetylcysteine (NAC) infusion was started in Emergency for managing liver toxicity. The patient was administered intravenous NAC with initial loading dose of $150 \mathrm{mg} / \mathrm{kg}$ over 1 hour, followed by $12.5 \mathrm{mg} / \mathrm{kg} / \mathrm{h}$ for 4 hours and continuous infusion of $6.25 \mathrm{mg} / \mathrm{kg} / \mathrm{h}$ for remaining 67 hours. In absence of specific antidote, patient required Liver transplant on day 7 of admission, and gradually patient's liver enzymes and bilirubin levels returned to normal levels. Patient was extubated after 15 days. Patient recovered gradually. He was discharged after one month of surgery. He didn't had any further episode of seizure or jaundice later after 2 months of liver transplant surgery and returned to his routine studies in school.

\section{Case Discussion}

Excessive Aluminium intake is seen in individuals who ingest large quantities of Aluminium containing antacids, or who are on phosphate binding therapy, or on regular dialysis. Increased Aluminium intake may also occur through certain vegetables (especially spinach), water boiled in Aluminium vessels and certain IV solutions. Cooking in Aluminium cookware also can raise the intake, as also packaging food in Aluminium foils. Soluble forms of Aluminium (such as Aluminium chloride, Aluminium sulfate. Aluminium fluoride and Aluminium citrate) have greater potential for toxicity, than insoluble forms such as Aluminium hydroxide [15]. On oral consumption of Alum, patient can have burning sensation in mouth, throat and abdomen as alum acts as a corrosive agent locally. The initial symptoms are vomiting with a fast pulse, hypothermia and shock. Convulsions may occur on acute overdose, especially in children in their teens [2]. Supportive treatment is indicated in form of protection of airway, controlling the seizures and prevention of further toxicity to vital organs. Ingested aluminum effect several biochemical functions, including neurotransmitter manufacture, uptake, and release. Chronic Aluminum toxicity can lead to encephalopathy characterized by seizures, myoclonus, and dementia, to osteomalacia, and to microcytic anemia. Management of chronic aluminum toxicity centers on removal from the source of exposure. The only chelator with proven benefit is deferoxamine which has a high affinity for aluminum and forms a dialyzable aluminum-deferoxamine complex. But deferoxamine is an orphan drug, not available commercially in most Indian pharmacies for patients. If chronic aluminium toxicity is detected early, successful treatment with full recovery of neurologic function has been reported with deferoxamine (DFO). DFO binds aluminum ions to form aluminoxamine, which is excreted renally. In patients with renal insufficiency, hemodialysis (especially with a high flux membrane) is effective in removing the aluminoxamine. The dosing of DFO should be tailored to the patient's serum Aluminium concentrations, symptomatology, and response. Doses of DFO of $15 \mathrm{mg} /$ $\mathrm{kg} /$ day infused over 1 hour at before hemodialysis have maximized aluminoxamine removal.

Acute Toxic dose of ALUM: 15-30 gm of alum may be fatal [2].

Fatalities from Alum poisoning are uncommon, but have been reported. One Drachm given in syrup killed the child, who was suffering from diphtheria [3]. A woman died after ingesting $5 \mathrm{~g}$ of Alum powder dissolved in water. She developed a high anion gap metabolic acidosis, multiple organ failure including ARDS and refractory shock [16]. Mild gastrointestinal symptoms were reported in Camelford (England) in 1988 after residents drank water to which Alum had inadvertently been added in substantial amount. Although there are no confirmed cases of ongoing adverse sequelae, there have been reports of long term cognitive impairment, such as short term memory loss and impaired concentration, in some of those exposed to chronic Alum intake. One of those exposed developed a cerebral angiopathy and died although the link with aluminium exposure was not proven [17].

There is no specific laboratory test mentioned in literature to detect serum Alum levels, but serial serum aluminium levels can guide on toxic exposure of Alum and monitoring of critical state of patient. In toxic doses of ALUM consumption, it may rarely lead to 
Fulminant Hepatic failure. Fulminant hepatic failure (FHF), also known as acute liver failure, is a rare condition resulting in rapid deterioration of liver function often followed by a cascade of devastating consequences. The mortality rate of FHF is as high as 40$50 \%$, depending on the cause and therapeutic management. The syndrome of FHF is incited by a catastrophic insult to the liver in an otherwise healthy individual. This liver injury can result in sudden onset of hepatic encephalopathy, often in association with coagulopathy, jaundice, and multisystem organ failure. FHF is a true medical emergency and carries a very high mortality rate.

It is well studied and known that N-Acetyl Cysteine (NAC) when given within the first $24 \mathrm{~h}$ after toxic overdose of any hepatotoxins (including Paracetamol, Aflatoxin etc.,) can prevent or minimize liver damage, by improving systemic hemodynamics, tissue oxygen delivery, and other favorable effects on the acutely injured liver. Nausea and vomiting were the symptoms more frequent during treatment with NAC. Along with its excellent safety profile, NAC is easy to administer, does not require intensive care monitoring, and can be given in community hospitals [13].

An improvement in the morbidity and mortality outcomes associated with FHF has not been seen until recently with advanced understanding, intensive medical therapy, and monitoring and the use of orthotopic liver transplant. The general management of a patient with FHF includes ensuring the patient is being cared for in an intensive care setting at a center with an active liver transplantation program, monitoring for worsening liver failure, treating complications, and providing nutritional support [11]. The term "fulminant hepatic failure" was first introduced more than 30 years ago by Trey., et al. to describe the onset of altered mental status within 8 weeks of initial symptoms in an individual with no previous history of liver disease [12]. Patients with FHF are particularly vulnerable to infection, bleeding, and cerebral edema.

\section{Conclusion}

Fulminant hepatic failure (FHF) due to ALUM toxicity is a very challenging and serious medical condition. It tests our best clinical and surgical skills because of its rarity, rapid progression, and frequently poor outcomes. Early identification of FHF and the administration of etiology-specific treatment are crucial in its management. Rescue therapies by administering N- Acetyl cysteine may provide temporary liver support in acute toxicity. Deferoxamine can be used as chelating agent for chronic exposure of aluminium and should be commercially available for therapy. The liver transplantation has reduced the mortality rate associated with FHF in youth.

\section{Conflict of Interest}

None declared.

\section{Bibliography}

1. Modi's Textbook of Forensic Medicine and Toxicology, 6th Ed. (1946): 345.

2. "Textbook on Diagnosis and Management of Common Poisoning". Dr Praveen Aggarwal, Jyoti Prakash Wali. Phitkari Poisoning. Oxford University Press, 1st Ed. (1997): 481.

3. “Taylor's Principle \& Practice of Medical Jurisprudence." 9th Ed. Churchill 2 (1865): 19.

4. Bharathi., et al. "Molecular toxicity of aluminium in relation to neurodegeneration" Indian Journal of Medical Research 128.4 (2008): 545-556.

5. M Kawahara., et al. "Effects of aluminum on the neurotoxicity of primary cultured neurons and on the aggregation of b-amyloid protein". Brain Research Bulletin 55.2 (2001): 211-217.

6. V Kumar and KD Gill "Oxidative stress and mitochondrial dysfunction in aluminium neurotoxicity and its amelioration: a review". Neurotoxicology 41 (2014): 154-166.

7. Fulgenzi A., et al. "Aluminium involvement in neurotoxicity". BioMed Research International (2014): 1-5.

8. M Nampoothiri., et al. "Modulatory role of simvastatin against aluminium chloride-induced behavioural and biochemical changes in rats". Behavioural Neurology (2015): 1-9.

9. Ogueche PN. "Aluminum Intoxication Induced Biochemical and Histopathological Alterations in Male Wistar Albino Rats Hepatocytes". Journal of Natural Sciences Research 4.24 (2014).

10. Hasan TÜRKEZ., et al. "The protective effect of boric acid on aluminum-induced hepatotoxicity and genotoxicity in rats". Turkish Journal of Biology 35.3 (2011): 293-301.

11. DL Halegoua-De Marzio and D. Sass. Textbook on Contemporary Liver Transplantation.

12. Sass DA and Shakil AO. "Fulminant hepatic failure". Gastroenterology Clinics of North America 32 (2003):1195-1211. 
13. Nabi T., et al. "Role of $\mathrm{N}$-acetylcysteine treatment in non-acetaminophen-induced acute liver failure: A prospective study". Saudi Journal of Gastroenterology 23.3 (2017): 169-175.

14. Prashant Verma and Chaitanya Namdeo."Treatment of Pediculosis Capitis". Indian Journal of Dermatology 60.3 (2015): 238-247.

15. Pillay VV. Comprehensive Medical Toxicology. 3rd Ed. Paras publishers. Hyderabad. (2018): 187-189.

16. Kim WJ., et al. "A case of 'Alum' intoxication leading to ARDS". Tuberculosis and Respiratory Diseases 53 (2002): 234-237.

17. Exley C and Esiri MM. "Severe cerebral conglophilic angiopathy coincident with increased brain aluminum in a resident of Camelford, cornwall, UK". Journal of Neurology, Neurosurgery, and Psychiatry 77.7 (2006): 877-879.

Volume 3 Issue 6 June 2019

(C) All rights are reserved by Vivekanshu Verma., et al. 